\title{
Lehman's Theorem and the Directed Steiner Tree Problem
}

\author{
Ahmad Abdi*, Andreas Emil Feldmann ${ }^{* *}$, Bertrand Guenin*, Jochen Könemann*, and \\ Laura Sanitä \\ * Department of Combinatorics and Optimization, University of Waterloo \\ \{a3abdi, bguenin, jochen, lsanita\}@uwaterloo.ca \\ ** Institute for Computer Science and Control, Hungarian Academy of Sciences (MTA SZTAKI) \\ feldmann.a.e@gmail.com
}

\begin{abstract}
In the directed Steiner tree problem, we are given a digraph, non-negative arc weights, a subset of vertices called terminals, and a special terminal called the root. The goal is to compute a minimum weight directed tree that connects each terminal to the root. We study the classical directed cut linear programming (LP) formulation which has a variable for every arc, and a constraint for every cut that separates a terminal from the root.

For what instances is the directed cut LP integral? In this paper we demonstrate how the celebrated theorem of Lehman [DIMACS, 1990] on minimally non-ideal clutters provides a framework for deriving answers to this question. Specifically, we show that this framework yields short proofs of the optimum arborescences theorem and the integrality result for series-parallel digraphs. Furthermore, we use this framework to show that the directed cut LP is integral for digraphs that are acyclic and have at most two non-terminal vertices.
\end{abstract}

\section{Introduction}

In the directed Steiner tree problem we are given a directed graph (digraph) $D=(V, A)$ with non-negative arc weights $w \in \mathbb{R}_{+}^{A}$, and a non-empty subset of terminals $R \subseteq V$ with a special vertex $r \in R$ called the root. A Steiner tree is an (inclusion-wise) minimal arc subset $T \subseteq A$ such that for each terminal $v \in R, T$ contains a $v r$-dipath. Vertices in $V-R^{1}$ are called Steiner vertices and need not be in $T$. The directed Steiner tree problem asks for a minimum weight Steiner tree. In this paper, we use a celebrated theorem of Lehman [24] to study the integrality of a natural linear programming (LP) relaxation of the directed Steiner tree problem. Moving forward, we will assume basic knowledge of polyhedral theory as well as graph theory; see the books of Conforti, Cornuéjols and Zambelli [5] and Bondy and Murty [1] for reference.

The standard directed cut LP formulation for the problem introduces a variable for each arc and imposes connectivity constraints for cuts separating a terminal from the root. For $U \subseteq V$ denote by $\delta_{D}^{+}(U)$ the set $\{(u, v) \in A: u \in U, v \in V-U\}$ of $\operatorname{arcs}$ exiting $U$. (We will omit the

\footnotetext{
${ }^{1}$ Given sets $A, B, A-B$ denotes the set $\{a \in A: a \notin B\}$.
} 
subscript $D$ whenever there is no ambiguity.) Let us call an arc subset $C \subseteq A$ a Steiner cut if $C$ is a minimal set of the form $\delta^{+}(U)$ for some $U \subseteq V-\{r\}$ such that $U \cap R \neq \emptyset$. If $\mathscr{C}$ denotes the family of all Steiner cuts, the directed Steiner tree problem can be formulated as the following integer programming problem:

$$
\begin{array}{cl}
\min & w^{\top} x \\
\text { s.t. } & \sum_{a \in C} x_{a} \geq 1, \quad \forall C \in \mathscr{C} \\
& x \in\{0,1\}^{A} .
\end{array}
$$

Consider the polyhedron obtained from (1) by replacing the integrality constraints with nonnegativity constraints, i.e. the polyhedron

$$
Q:=\left\{x \in \mathbb{R}_{+}^{A}: \sum_{a \in C} x_{a} \geq 1, \forall C \in \mathscr{C}\right\} .
$$

We will focus on sufficient conditions for $Q$ to be integral (i.e. for each face to contain an integer point). To state our main theorem we need two definitions. The underlying graph of a digraph $D$ is the graph obtained by ignoring the directions of the arcs and by replacing each maximal set of parallel edges by a single edge. A digraph is series-parallel if the underlying graph has no minor isomorphic to the complete graph $K_{4}$ on four vertices. ${ }^{2}$

Theorem 1. Let $D=(V, A)$ be a digraph with terminals $R$ and root $r$ and let $\mathscr{C}$ be the family of Steiner cuts. The polyhedron given in (2) is integral if any of the following conditions holds:

(i) $|R|=2$ (Ford and Fulkerson [12]),

(ii) $R=V$ (Edmonds [10], Fulkerson [15]),

(iii) D has no directed cycle and $|V-R| \leq 2$,

(iv) D is series-parallel (Prodon et al. [26], Schaffers [28], Goemans [16]).

In this paper, we provide a common framework based on Lehman's theorem on minimally non-ideal clutters with which one can unify the well-known results (i) (the shortest st-dipath problem), (ii) (the optimum arborescence problem) and (iv), and find short proofs for them, especially for (iv). To show its potential, we leverage Lehman's theorem and obtain the new result (iii). In fact, (iii) will appear as a special case of a more general result.

Each of (i)-(iv) in Theorem 1 is in a sense best possible. For instance, consider the digraphs $D_{1}, D_{2}$ and $D_{3}$ depicted in Figure 1. For $j \in[3]^{3}$ let $Q_{j}$ denote the polyhedron corresponding to $D_{j}$. Let us show that $Q_{j}$ is not integral. Define $\bar{x} \in Q_{j}$ where $\bar{x}_{1}=\bar{x}_{2}=\bar{x}_{3}=\frac{1}{2}$ and all other entries are equal to 1 (the indices for vector $\bar{x}$ correspond to the arc labels in the figure for $D_{j}$ ). Define a vector $w$ with the same number of components as $\bar{x}$ where $w_{1}=w_{2}=w_{3}=1$ and all other entries are equal to 0 . Since every Steiner tree of $D_{j}$ has to contain at least two edges from

\footnotetext{
${ }^{2}$ There is another way to define such digraphs that is more fitting with the terminology; see [9].
}

${ }^{3}[m]$ denotes the set $\{1, \ldots, m\}$ 


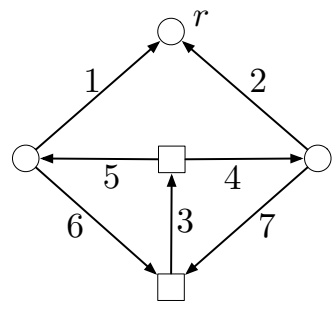

$D_{1}$

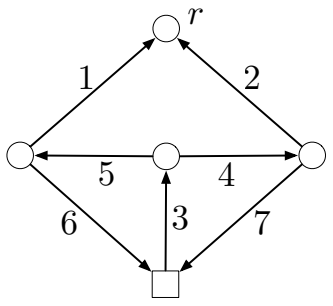

$D_{2}$

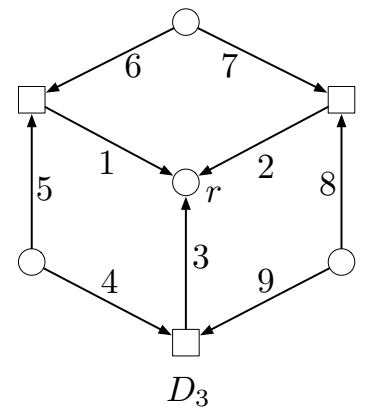

Figure 1: Round vertices are terminals, squares are Steiner vertices.

the edge set [3], $\min \left\{w^{\top} x: x \in Q_{j} ; x\right.$ integer $\} \geq 2$. Because $\bar{x} \in Q_{j}, \min \left\{w^{\top} x: x \in Q_{j}\right\} \leq \frac{3}{2}$. It follows that $Q_{j}$ is not integral.

Digraph $D_{1}$ has three terminals so (i) is tight, while $D_{2}$ has one Steiner vertex so (ii) is tight. For (iii) the condition that the digraph be acyclic cannot be omitted as $D_{1}$ (resp. $D_{2}$ ) has a directed cycle but $|V-R| \leq 2$. Furthermore, subject to the condition that the digraph is acyclic, (iii) is tight since for $D_{3},|V-R|=3$. Finally, for (iv) each of $D_{1}, D_{2}, D_{3}$ show that the condition that the digraph be series-parallel cannot be omitted.

\subsection{Related work and outline}

Our work is not the first one to use Lehman's theorem to obtain certain polyhedral descriptions. Shepherd [33] used this result to provide a polyhedral description for the convex hull of stable sets of near-bipartite graphs - graphs for which the deletion of any vertex produces a bipartite graph. As another application, the third author [17] used Lehman's theorem to provide an excluded minor characterization of weakly bipartite graphs - graphs for which the convex hull of bipartite subgraphs is defined by enforcing non-negativity constraints $x \geq \mathbf{0}$ and odd cycle inequalities $x(C) \leq|C|-1$.

Here we apply Lehman's theorem to the directed Steiner tree problem, a fundamental NP-hard network design problem that generalizes several classical optimization problems such as the setcover and group-Steiner tree problems. The latter cannot be approximated within $O\left(\log ^{2-\epsilon}(n)\right)$ for any $\epsilon>0$ unless NP-complete problems have quasi-polynomial time algorithms [18], and so neither can the directed Steiner tree problem. The problem has an $O\left(\log ^{3}(n)\right)$-approximation algorithm that runs in quasi-polynomial time [3]. The formulation we study in this paper is very natural, and therefore its relaxation has been widely investigated in the network design community. Zosin and Khuller [35] demonstrated that its integrality gap in general graphs can be as bad as $\Omega(\sqrt{k})$, where $k$ is the number of terminals. Friggstad et al. [13] showed that the integrality gap can be reduced to $O(\ell \cdot \log k)$ for (so called) $\ell$-layered graphs, by applying $\ell$ rounds of Sherali-Adams hierarchy [34] or $2 \ell$ rounds of Lovász-Schrijver hierarchy [25]. Rothvoß [27] has provided a similar result using Lasserre semidefinite programming hierarchy $[20,21]$.

In $\S 2$ we state Lehman's theorem and prove some necessary consequences. In $\S 3$ we restate 
our main theorem in the language of Lehman's theorem, and in $\S 4$ we prove the restated version. Finally in $\S 5$ we provide some evidence suggesting that a complete characterization of when the corresponding polyhedron (2) is integral is likely to remain elusive.

\section{Lehman's theorem and consequences}

We will use Lehman's powerful and very general result to prove Theorem 1. Vaguely speaking, this result finds a nice substructure whenever a generic LP, such as directed cut, allows for fractional extreme points. Here we state the result and in $\S 3$ we restate our main theorem in terms of fractional Steiner tree instances. This will allow us to use Lehman's theorem to prove none of the conditions (i)-(iv) can hold for fractional instances, due to a highly restrictive substructure.

To state Lehman's theorem we require a few definitions. Let $E$ be a finite set, called a ground set, and let $\mathscr{C}$ be a family of subsets of $E$, called members. We say $\mathscr{C}$ is a clutter over ground set $E=E(\mathscr{C})$ if no member is contained in, or is equal to, another member. A cover is a subset of $E$ that intersects every member of $\mathscr{C} .{ }^{4}$ For instance, for vertices $s$ and $t$ of a graph, the family of $s t$-paths is a clutter, an st-cut is a cover for this clutter, and every cover contains an st-cut. The blocker of $\mathscr{C}$, denoted $b(\mathscr{C})$, is another clutter over the same ground set whose members are the (inclusionwise) minimal covers. It can be shown that $b(b(\mathscr{C}))=\mathscr{C}[11,19]$. With clutter $\mathscr{C}$ we associate the polyhedron

$$
Q(\mathscr{C})=\left\{x \in \mathbb{R}_{+}^{E(\mathscr{C})}: \sum_{e \in C} x_{e} \geq 1, \forall C \in \mathscr{C}\right\} .
$$

Observe that the integer extreme points of $Q(\mathscr{C})$ are precisely the characteristic vectors of minimal covers. A clutter $\mathscr{C}$ is ideal if the polyhedron is integral, otherwise it is non-ideal. For example, the clutter $\{\{1,2\},\{2,3\},\{3,1\}\}$ is non-ideal as its associated polyhedron has $\left(\frac{1}{2}, \frac{1}{2}, \frac{1}{2}\right)^{\top}$ as an extreme point. It is known that if a clutter is ideal, then so is its blocker [14, 23].

One can define two minor operations on $\mathscr{C}$. The contraction $\mathscr{C} / e$ of an element $e \in E$ is the clutter over the ground set $E-\{e\}$ consisting of the minimal sets in $\{C-\{e\}: C \in \mathscr{C}\}$. That is, for $\mathscr{C} / e$ the element $e$ is removed from each member of $\mathscr{C}$. The deletion $\mathscr{C} \backslash e$ is the clutter over the ground set $E-\{e\}$ with members $\{C: C \in \mathscr{C}, e \notin C\}$. That is, for $\mathscr{C} \backslash e$ those members of $\mathscr{C}$ containing $e$ are removed. It is known that $b(\mathscr{C} / e)=b(\mathscr{C}) \backslash e$ and $b(\mathscr{C} \backslash e)=b(\mathscr{C}) / e[31]$. A clutter $\mathscr{C}^{\prime}$ obtained from a clutter $\mathscr{C}$ by deleting elements $I \subseteq E$ and contracting elements $J \subseteq E$ is a minor of $\mathscr{C}$ (and $\mathscr{C}^{\prime}$ does not depend on the order of the operations, that is, minor operations commute). We denote $\mathscr{C}^{\prime}$ by $\mathscr{C} / J \backslash I$. The clutter $\mathscr{C}^{\prime}$ is a proper minor of $\mathscr{C}$ if $I \cup J \neq \emptyset$. If a clutter is ideal, then so are all its minors [32]. A clutter is minimally non-ideal (mni) if it is non-ideal and all of its proper minors are ideal. For an integer $k \geq 2$, a clutter is a degenerate projective plane if, after possibly relabeling its elements, it has ground set $\{0,1, \ldots, k\}$ and members $\{\{1, \ldots, k\},\{0,1\},\{0,2\}, \ldots,\{0, k\}\}$. Every degenerate projective plane is mni [23].

\footnotetext{
${ }^{4}$ Our terminology of the term "cover" closely follows Schrijver [29]; he refers to "covers" as "vertex covers". In the literature, covers have also been referred to as "transversals", "hitting sets" as well as "blocking sets".
} 
With clutter $\mathscr{C}$ we associate a 0,1 matrix $M(\mathscr{C})$ whose columns are indexed by $E$ and whose rows are the incidence vectors of the members of $\mathscr{C}$. Note that $M(\mathscr{C})$ is defined up to permutations of the rows (not the columns, though). A square 0,1 matrix is $r$-regular if each row and each column has $r$ ones. Given a clutter $\mathscr{C}$ we denote by $\overline{\mathscr{C}}$ the clutter that consists of all sets of $\mathscr{C}$ of minimum cardinality.

Theorem 2 (Lehman [24], also see Seymour [30]).

Let $\mathscr{C}$ be an mni clutter that is not a degenerate projective plane and let $\mathscr{T}=b(\mathscr{C})$. Then

(1) $M(\overline{\mathscr{C}})$ and $M(\overline{\mathscr{T}})$ are square and non-singular,

(2) for some integers $c \geq 2$ and $t \geq 2: M(\overline{\mathscr{C}})$ is c-regular and $M(\overline{\mathscr{T}})$ is t-regular,

(3) the rows of $M(\overline{\mathscr{C}})$ can be permuted so that

$$
M(\overline{\mathscr{C}}) M(\overline{\mathscr{T}})^{\top}=J+d I^{5}
$$

where $d=c t-|E(\mathscr{C})| \geq 1$.

Lehman also proved that if $\mathscr{C}$ is mni, then $Q(\mathscr{C})$ has a unique fractional extreme point. When $\mathscr{C}$ is not a degenerate projective plane then $\left(\frac{1}{c}, \ldots, \frac{1}{c}\right)^{\top}$ is the fractional extreme point of $Q(\mathscr{C})$.

The matrices in equation $(\star)$ of Theorem 2 commute, as the following result shows.

Theorem 3 (Bridges and Ryser [2]).

Let $A, B$ be square 0,1 matrices where $A B=J+d I$ for some $d \geq 1$. Then $A B=B A$.

A transversal of a clutter $\mathscr{C}$ is a subset of the ground set that intersects every member of $\mathscr{C}$ in exactly one element. ${ }^{6}$ Let $\mathscr{C}$ be an mni clutter. If $\mathscr{C}$ is a degenerate projective plane let $\operatorname{core}(\mathscr{C})=\mathscr{C}$, otherwise let $\operatorname{core}(\mathscr{C})=\overline{\mathscr{C}}$. The following proposition lists well-known corollaries of Lehman's theorem. We include proofs for completeness.

Proposition 4 (Cornuéjols et al. [6], Guenin [17]).

Let $\mathscr{C}$ be an mni clutter and let $\mathscr{T}=b(\mathscr{C})$ and $m=|E(\mathscr{C})|$. Then core $(\mathscr{C})$ has $m$ members, say $C_{1}, \ldots, C_{m}$ and core $(\mathscr{T})$ has $m$ members, say $T_{1}, \ldots, T_{m}$. After possibly relabeling $C_{1}, \ldots, C_{m}$ the following hold:

(F1) for $i, j \in[m],\left|C_{i}\right| \geq 2,\left|T_{j}\right| \geq 2$ and

$$
\left|C_{i} \cap T_{j}\right| \quad \begin{cases}>1 & \text { if } i=j \\ =1 & \text { if } i \neq j,\end{cases}
$$

(F2) for distinct $a, b \in E(\mathscr{C})$, there exist $i, j \in[m]$ such that $a \in C_{i}, b \notin C_{i}$ and $a \in T_{j}, b \notin T_{j}$,

\footnotetext{
${ }^{5} J$ denotes the matrix of all ones and $I$ the identity matrix.

${ }^{6}$ Once again, our terminology closely follows Schrijver [29].
} 
(F3) for $a \in E(\mathscr{C})$, there exist distinct $i, j \in[m]$ such that,

$$
C_{i} \cap C_{j}=T_{i} \cap T_{j}=C_{i} \cap T_{j}=C_{j} \cap T_{i}=\{a\}
$$

(F4) there is no transversal of core $(\mathscr{C})$ (resp. core $(\mathscr{T})$ ).

Proof. We assume $\mathscr{C}$ is not a degenerate projective plane, for otherwise $\mathscr{T}=\mathscr{C}$ and we leave it as an easy exercise to verify that (F1)-(F4) hold. Relabel $C_{1}, \ldots, C_{m}$ so that equation ( $\star$ ) in Theorem 2 holds. (F1) follows from $(\star)$. (F2) By Theorem 2(1) $M(\overline{\mathscr{C}})$ is non-singular, in particular columns $a$ and $b$ of $M(\overline{\mathscr{C}})$ are distinct. By Theorem 2(2) columns $a$ and $b$ of $M(\overline{\mathscr{C}})$ both have exactly $c$ entries with a 1 . Hence, there is a row $C_{i}$ with $a \in C_{i}, b \notin C_{i}$. Applying the same argument to $M(\overline{\mathscr{T}})$ we obtain $T_{j}$. (F3) Equation $(\star)$, together with Theorem 3, implies that $J+d I=M(\overline{\mathscr{C}}) M(\overline{\mathscr{T}})^{\top}=M(\overline{\mathscr{C}})^{\top} M(\overline{\mathscr{T}})$. This implies that for all $e, f \in E(\mathscr{C})$,

$$
\operatorname{col}_{e}(M(\overline{\mathscr{C}})) \cdot \operatorname{col}_{f}(M(\overline{\mathscr{T}})) \quad \begin{cases}>1 & \text { if } e=f \\ =1 & \text { if } e \neq f\end{cases}
$$

where denotes the dot product. Choosing $e=f=a$ in $(\dagger)$ implies that there exist $i, j \in[m]$ such that $\{a\} \in C_{i} \cap C_{j} \cap T_{i} \cap T_{j}$. Choosing $f=a$ and all $e \in C_{i} \cup C_{j}-\{a\}$ in (†) implies that $C_{i} \cap C_{j}=\{a\}$. Choosing $e=a$ and all $f \in T_{i} \cup T_{j}-\{a\}$ in (†) implies that $T_{i} \cap T_{j}=\{a\}$. Finally, by (F1), $C_{i} \cap T_{j}=C_{j} \cap T_{i}=\{a\}$. (F4) Suppose for a contradiction there exists a transversal $S$ of $\overline{\mathscr{C}}$ and let $\chi_{S}$ denote the characteristic vector of $S$. Then $M(\overline{\mathscr{C}}) \chi_{S}=\mathbf{1}$. By Theorem $2(2)$ $M(\overline{\mathscr{C}})$ is $c$-regular, so $M(\overline{\mathscr{C}})\left(\frac{1}{c}, \ldots, \frac{1}{c}\right)^{\top}=\mathbf{1}$. Thus $M(\overline{\mathscr{C}}) x=\mathbf{1}$ has two distinct solutions, a contradiction as $M(\overline{\mathscr{C}})$ is non-singular, by Theorem $2(1)$. The proof for $\overline{\mathscr{T}}$ is identical.

\section{A restatement of the main theorem}

In Theorem 1 we are looking for sufficient conditions for the clutter $\mathscr{C}$ of Steiner cuts of a digraph to be ideal. If $\mathscr{C}$ is non-ideal then it has an mni minor $\mathscr{C}^{\prime}$. However, $\mathscr{C}^{\prime}$ need not be a clutter of Steiner cuts. For instance if $\mathscr{C}$ is the clutter of Steiner cuts of $D_{1}$ in Figure 1 then $\mathscr{C}^{\prime}=\mathscr{C} /\{4,5,6,7,8\}=\{\{1,2\},\{2,3\},\{1,3\}\}$ which is not the clutter of Steiner cuts of any digraph. Therefore, we need to extend the definitions of Steiner cuts and Steiner trees, so that their clutters form a minor closed class.

Let $D=(V, A)$ be a digraph with terminals $R$ and root $r$. A marked digraph is a pair $(D, \Gamma)$, where $\Gamma \subseteq A$ is a set of active arcs. We refer to $\operatorname{arcs}$ in $A-\Gamma$ as inactive arcs. A Steiner cut of $(D, \Gamma)$ is a Steiner cut of $D$ that does not contain an inactive arc. A subset of active $\operatorname{arcs} T$ is a Steiner tree of $(D, \Gamma)$ if $T \cup(A-\Gamma)$ contains a Steiner tree for $D$, and $T$ is minimal subject to this condition. If $\Gamma=A$ then our definitions of Steiner cut and Steiner tree reduce to the standard ones. Note that the ground set of the clutter of Steiner cuts (resp. Steiner trees) for a marked digraph is the set $\Gamma$ of active arcs.

Consider a marked digraph $(D, \Gamma)$ with an active arc $a$. The operation that consists of replacing $(D, \Gamma)$ by $(D, \Gamma-\{a\})$ is called inactivating $a$. 
Proposition 5. Let $(D, \Gamma)$ be a marked digraph with terminals $R$ and root $r$ where $D=(V, A)$. Let $\mathscr{C}$ be the clutter of Steiner cuts of $(D, \Gamma)$, let $\mathscr{T}$ be the clutter of Steiner trees of $(D, \Gamma)$ and let $a \in \Gamma$. Then

(1) $\mathscr{C} / a$ is the clutter of Steiner cuts of the marked digraph obtained by deleting a in $(D, \Gamma)$,

(2) $\mathscr{C} \backslash a$ is the clutter of Steiner cuts of the marked digraph obtained by inactivating a in $(D, \Gamma)$,

(3) $\mathscr{T} / a$ is the clutter of Steiner trees of the marked digraph obtained by inactivating a in $(D, \Gamma)$,

(4) $\mathscr{T} \backslash a$ is the clutter of Steiner trees of the marked digraph obtained by deleting a in $(D, \Gamma)$,

(5) $b(\mathscr{C})=\mathscr{T}$.

Proof. Parts (1)-(4) follow from the definition of minors. For (5), let $\mathscr{C}^{o}$ (resp. $\mathscr{T}^{o}$ ) be the clutter of Steiner cuts (resp. trees) of the digraph $D$. It is well known that $b\left(\mathscr{C}^{o}\right)=\mathscr{T}^{o}[4]$. (2) implies $\mathscr{C}=\mathscr{C}^{\circ} \backslash(A-\Gamma)$ and $(3)$ implies $\mathscr{T}=\mathscr{T}^{\circ} /(A-\Gamma)$. Hence, $b(\mathscr{C})=b\left(\mathscr{C}^{o} \backslash(A-\Gamma)\right)=$ $b\left(\mathscr{C}^{\circ}\right) /(A-\Gamma)=\mathscr{T}^{\circ} /(A-\Gamma)=\mathscr{T}$.

The following result will be proved in the next section.

Theorem 6. Let $(D=(V, A), \Gamma)$ be a marked digraph with terminals $R$ and root $r$, where the clutter of Steiner cuts $\mathscr{C}$ is mni. Then the following hold:

(i') $|R| \geq 3$,

(ii') $V-R \neq \emptyset$,

(iii') D has a directed cycle or

$$
|V-R| \geq \max \left\{t+1, t^{\prime}\right\}
$$

where $t$ (resp. $\left.t^{\prime}\right)$ is the cardinality of the smallest (resp. largest) Steiner tree.

(iv') $D$ is not series-parallel.

Note that in (iii'), $t \geq 2$ because of Theorem 2 . Hence in particular $|V-R| \geq 3$ when $D$ has no directed cycle. Assuming Theorem 6 we can now prove Theorem 1.

Proof of Theorem 1. We will prove the contrapositive statement. Let $\mathscr{C}$ be the clutter of Steiner cuts of $D=(V, A)$ and suppose it is non-ideal. Then $\mathscr{C}$ has an mni minor $\mathscr{C}^{\prime}$. By Proposition 5 , $\mathscr{C}^{\prime}$ is the clutter of a marked digraph $\left(D^{\prime}, \Gamma\right)$ obtained from $(D, A)$ by deleting and inactivating arcs. By Theorem 6, (i')-(iv') hold for $\left(D^{\prime}, \Gamma\right)$, implying that (i')-(iv') hold for $(D, A)$ as well this is because $D^{\prime}$ and $D$ have the same set of terminals and Steiner vertices, a directed cycle in $D^{\prime}$ corresponds to a directed cycle in $D$, and the underlying graph of $D^{\prime}$ is a deletion minor of the underlying graph of $D$. Hence, none of (i)-(iv) of Theorem 1 can hold for $D$, finishing the proof. 


\section{A proof of the restatement of the main theorem}

In this section we will prove Theorem 6 and hence complete the proof of our main result, Theorem 1. Throughout this section $(D=(V, A), \Gamma)$ is a marked digraph with terminals $R$ and root $r$ where the clutter of Steiner cuts $\mathscr{C}$ is mni. Let $\mathscr{T}=b(\mathscr{C})$. By Proposition $5, \mathscr{T}$ is the clutter of Steiner trees. We will assume that core $(\mathscr{C})$ has $m$ members $C_{1}, \ldots, C_{m}$, and that $\operatorname{core}(\mathscr{T})$ has $m$ members $T_{1}, \ldots, T_{m}$, and these members are labeled so that (F1)-(F4) of Proposition 4 hold. If $\mathscr{C}$ (resp. $\mathscr{T}$ ) is not a degenerate projective plane then, for some $c, t \geq 2$ and each $j \in[m]$, we have $\left|C_{j}\right|=c$ and $\left|T_{j}\right|=t$. For each $j \in[m]$, choose a minimal subset $U_{j} \subseteq V-\{r\}$ for which $\delta^{+}\left(U_{j}\right)=C_{j}$ and $U_{j} \cap R \neq \emptyset$.

\subsection{Parts $\left(i^{\prime}\right)$ and $\left(i i^{\prime}\right)$}

Two sets $U$ and $W$ are said to be laminar if either $U \cap W=\emptyset, U \subseteq W$ or $W \subseteq U$.

Lemma 7. For $i, j \in[m]$, either $U_{i}, U_{j}$ are laminar or $U_{i} \cap U_{j} \cap R=\emptyset$.

Proof. Consider first the case where $\mathscr{C}$ is not a degenerate projective plane. Suppose for a contradiction that $U_{i} \cap U_{j} \cap R \neq \emptyset$ and that $U_{i}, U_{j}$ are not laminar, i.e. that $U_{i}-U_{j}, U_{j}-U_{i} \neq \emptyset$. Let $U=U_{i} \cap U_{j}$ and let $W=U_{i} \cup U_{j}$. For a set $S \subseteq A$ we denote by $\chi_{S}$ the characteristic vector of $S$. It can be readily checked that,

$$
\chi_{\delta^{+}\left(U_{i}\right)}+\chi_{\delta^{+}\left(U_{j}\right)} \geq \chi_{\delta^{+}(U)}+\chi_{\delta^{+}(W)} .
$$

(In particular, $\delta^{+}(U), \delta^{+}(W)$ consist only of active arcs.) Recall that $\overline{\mathscr{C}}=\left\{\delta^{+}\left(U_{1}\right), \ldots, \delta^{+}\left(U_{m}\right)\right\}$ is the set of minimum Steiner cuts. By hypothesis, $\left|\delta^{+}\left(U_{i}\right)\right|=\left|\delta^{+}\left(U_{j}\right)\right|=c$ and since $\delta^{+}(U)$ and $\delta^{+}(W)$ are, or contain, Steiner cuts, $\left|\delta^{+}(U)\right|,\left|\delta^{+}(W)\right| \geq c$. Hence, $(\star \star)$ holds with equality and $\left|\delta^{+}(U)\right|=\left|\delta^{+}(W)\right|=c$. Thus for some $k, \ell \in[m]$ we have $\delta^{+}(U)=\delta^{+}\left(U_{k}\right)$ and $\delta^{+}(W)=\delta^{+}\left(U_{\ell}\right)$. Since $(\star \star)$ holds with equality, it follows that the zero vector is obtained from $M(\overline{\mathscr{C}})$ by adding rows $i, j$ and subtracting rows $k, \ell$. As $M(\overline{\mathscr{C}})$ is non-singular (see Theorem 2(1)), we must have $\{i, j\}=\{k, \ell\}$, say $i=k, j=\ell$. It follows that $\delta^{+}\left(U_{i}\right)=\delta^{+}\left(U_{k}\right)=\delta^{+}(U)=\delta^{+}\left(U_{i} \cap U_{j}\right)$. But by hypothesis $U_{i} \cap U_{j} \subset U_{i}$, contradicting the minimality of $U_{i}$. Consider now the case where $\mathscr{C}$ is a degenerate projective plane. Then $(\star \star)$ still holds. Moreover, it can be readily checked that in this case, the only possible choice is to have $\left\{\delta^{+}\left(U_{i}\right), \delta^{+}\left(U_{j}\right)\right\}=\left\{\delta^{+}(U), \delta^{+}(W)\right\}$. Then proceed as in the previous case.

Lemma 8. There exists a pair $i, j \in[m]$ such that $U_{i}, U_{j}$ are not laminar.

Proof. Choose a subset $U_{j}$ that is inclusionwise minimal among $U_{1}, U_{2}, \ldots, U_{m}$. By (F1) there exist distinct arcs $a, b \in \delta^{+}\left(U_{j}\right)$. Denote by $t_{a}$ and $h_{a}$ the tail and head of $a$ and by $t_{b}$ and $h_{b}$ the tail and head of $b$. By (F2) there exist indices $k, \ell \in[m]$ such that $a \in \delta^{+}\left(U_{k}\right) \not \supset b$ and $b \in \delta^{+}\left(U_{\ell}\right) \not \supset a$. We claim that either $U_{k}, U_{j}$ or $U_{\ell}, U_{j}$ are not laminar. Suppose otherwise. We have $t_{a} \in U_{j} \cap U_{k} \neq \emptyset$. Since $U_{j}, U_{k}$ are laminar and since $U_{j}$ is minimal, $U_{k} \supseteq U_{j}$. Similarly, $t_{b} \in U_{j} \cap U_{\ell}$ and $U_{\ell} \supseteq U_{j}$. Then $U_{k} \cap U_{\ell} \supseteq U_{j} \neq \emptyset$ and $h_{a} \in U_{\ell}-U_{k}, h_{b} \in U_{k}-U_{\ell}$, i.e. $U_{k}, U_{\ell}$ are not laminar. However, $U_{k} \cap U_{\ell} \cap R \supseteq U_{j} \cap R \neq \emptyset$, a contradiction with Lemma 7 . 
We are ready to prove Theorem 6 parts (i') and (ii'), stating that $|R| \geq 3$ and $V-R \neq \emptyset$.

Proof of Theorem 6, parts ( $\left.i^{\prime}\right)$ and (ii'). By Lemma 8, there exists a pair $i, j \in[m]$ where $U_{i}, U_{j}$ are not laminar. Lemma 7 implies that $U_{i} \cap U_{j} \cap R=\emptyset$. Thus $U_{i}-U_{j}$ and $U_{j}-U_{i}$ must each contain a terminal. Together with the root we thus have at least 3 terminals proving (i'). Finally, as $U_{i} \cap U_{j} \neq \emptyset$ it must contain a Steiner vertex, thus proving (ii').

\subsection{Part $\left(i i i^{\prime}\right)$}

For $U \subseteq V$, denote by $D[U]$ the subdigraph of $D$ with arcs having both tail and head in $U$.

Remark 9. Take $j \in[m]$ and $u, v \in U_{j}$ with $u \in R$. Then there exists a uv-dipath in $D\left[U_{j}\right]$.

Proof. For otherwise let $U_{j}^{\prime}$ contain $u$ as well as all vertices $w$ for which there exists a $u w$-dipath in $D\left[U_{j}\right]$. Then $\delta^{+}\left(U_{j}^{\prime}\right) \subseteq \delta^{+}\left(U_{j}\right), u \in U_{j}^{\prime} \cap R \neq \emptyset$ and $U_{j}^{\prime} \subseteq U_{j}-\{v\}$, contradicting the minimality of $\delta^{+}\left(U_{j}\right)$ or $U_{j}$.

We are ready to prove Theorem 6 part (iii'), claiming that either $D$ is not acylic or $|V-R| \geq$ $\max \left\{t+1, t^{\prime}\right\}$ where $t$ (resp. $t^{\prime}$ ) is the cardinality of the smallest (resp. largest) Steiner tree.

Proof of Theorem 6, part (iii'). Suppose that $D$ is acyclic.

Claim. The tail of every active arc is a Steiner vertex.

Proof. Suppose for a contradiction that the tail $t_{a}$ of some active $\operatorname{arc} a$ is a terminal. Because of (F3) there exist $i, j \in[m]$ such that,

$$
C_{i} \cap C_{j}=T_{i} \cap C_{j}=\{a\} .
$$

Since $t_{a} \in U_{i} \cap U_{j} \cap R$, Lemma 7 implies that $U_{i}, U_{j}$ are laminar. Thus we may assume $U_{i} \subseteq U_{j}$. By (F1) there exists $b \in\left(C_{i} \cap T_{i}\right)-\{a\}$. Denote by $t_{b}$ the tail of $b$ and by $h_{b}$ the head of $b$. Then $t_{b} \in U_{i}$ and $h_{b} \notin U_{i}$. By $(\dagger \dagger) b \notin C_{j}$, hence $h_{b}=U_{j}-U_{i}$. By Remark 9 there exists a $t_{a} t_{b}$-dipath $P$ in $D\left[U_{i}\right]$. Since $b \in T_{i}$, there exists a dipath from $h_{b}$ to the root $r$ in $T_{i} \cup(A-\Gamma)$. It follows from ( $\dagger \dagger$ ) that this dipath must contain $a$. Therefore, there exists an $h_{b} t_{a}$-dipath $Q$. But then $P \cup Q \cup\{b\}$ contains a directed circuit, a contradiction.

For each Steiner tree $T$, let $W(T)$ collect the arc tails of $T$. It follows from Claim that all vertices in $W(T)$ are Steiner vertices. Since $T$ is contained in a tree of $D$ rooted towards $r$, no two arcs of $T$ share a tail, so $|W(T)|=|T|$. Hence, any Steiner tree $T$ implies that we have at least $|T|$ Steiner vertices, so $|V-R| \geq t^{\prime}$. We may assume that there is no Steiner tree $T$ of cardinality greater than $t$ (the minimum cardinality of a Steiner tree) for otherwise we are done. Suppose, for a contradiction, that we have exactly $t$ Steiner vertices. Then for all $i, j \in[m], W\left(T_{i}\right)=W\left(T_{j}\right)$. Pick $w \in W\left(T_{1}\right)$. Then $\delta^{+}(w) \cap \Gamma$ is a transversal of $T_{1}, \ldots, T_{m}$, contradicting (F4). 


\subsection{Part $\left(i v^{\prime}\right)$}

Let $(D, \Gamma)$ be a marked digraph with terminals $R$ and root $r$. We say that the 4-tuple $(D, \Gamma, R, r)$ is a representation of a clutter $\mathscr{C}$ if $\mathscr{C}$ is the clutter of Steiner cuts of $(D, \Gamma)$ with terminals $R$ and root $r$. It is possible for a clutter of Steiner cuts to have several representations and it will be important for the proof to select a suitable representation. For $j=1,2$ consider a marked digraph $\left(D_{j}, \Gamma_{j}\right)$ with terminals $R_{j}$ and root $r_{j}$. We say that $\left(D_{1}, \Gamma_{1}, R_{1}, r_{1}\right)$ is reducible to $\left(D_{2}, \Gamma_{2}, R_{2}, r_{2}\right)$ if $\left(D_{1}, \Gamma_{1}, R_{1}, r_{1}\right)$ and $\left(D_{2}, \Gamma_{2}, R_{2}, r_{2}\right)$ are representations of the same clutter, and the underlying graph of $D_{2}$ is a proper minor of (an isomorphic ${ }^{7}$ copy of) the underlying graph of $D_{1}$. (See Figure 2 for an example.) A representation $(D, \Gamma, R, r)$ is irreducible if it is not reducible to any other representation.

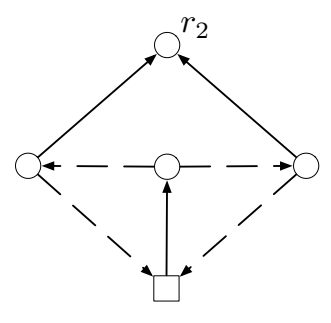

$\left(D_{2}, \Gamma_{2}, R_{2}, r_{2}\right)$

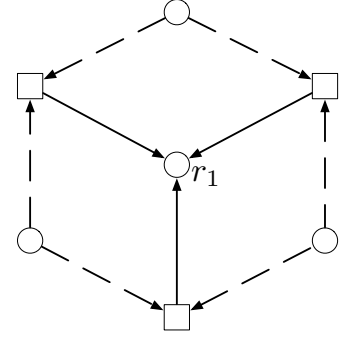

$\left(D_{1}, \Gamma_{1}, R_{1}, r_{1}\right)$

Figure 2: Two representations of $\{\{1,2\},\{2,3\},\{3,1\}\}$ where $\left(D_{1}, \Gamma_{1}, R_{1}, r_{1}\right)$ reduces to $\left(D_{2}, \Gamma_{2}, R_{2}, r_{2}\right)$. Round vertices are terminals, solid arcs are active.

Let $G$ be a graph, let $U \subseteq V(G)$ and if $U \neq \emptyset$, take $u_{0} \in U$. Consider the following properties:

(P1) $G$ is a simple graph and $V(G) \neq \emptyset$,

(P2) vertices in $U$ have degree two, other vertices have degree at least three,

(P3) $U$ is an independent set,

(P4) the two neighbours of any $u \in U-\left\{u_{0}\right\}$ are non-adjacent,

(P5) no two vertices in $U-\left\{u_{0}\right\}$ have the same set of neighbours.

Lemma 10. If a graph $G$ satisfies properties (P1)-(P5) for some $U \subseteq V(G)$, then $G$ has a $K_{4}$ minor.

Proof. We may assume that $G$ is minor minimal with respect to satisfying (P1)-(P5), i.e. it satisfies all these properties, but no proper minor of $G$ does. Let $U$ be the set of vertices of degree two of $G$. We claim that $U=\emptyset$ or $\left\{u_{0}\right\}$ where $u_{0}$ has adjacent neighbours. For if $u \in U$ has non-adjacent neighbours $v_{1}$ and $v_{2}$, we can replace edges $v_{1} u$ and $u v_{2}$ by an edge $v_{1} v_{2}$ without creating parallel edges. We leave it as an exercise to check that (P1)-(P5) hold for the resulting graph, contradicting the minimality of $G$.

\footnotetext{
${ }^{7}$ we are allowed to first relabel the edges
} 
We next claim that $G$ is 2-connected. If $G$ has distinct components, then each component will satisfy properties (P1)-(P5), contradicting the minimality of $G$. Hence, $G$ is connected. Suppose that $G$ has a cut vertex $v$ and let $G_{1}$ and $G_{2}$ be the induced subgraphs of $G$ with $V\left(G_{1}\right) \cap V\left(G_{2}\right)=\{v\}$ and $V\left(G_{1}\right) \cup V\left(G_{2}\right)=V(G)$. We may assume that if $U \neq \emptyset$, then the unique vertex $u_{0} \in U$ is in $V\left(G_{2}\right)$. We may also assume, by choosing the cut vertex appropriately, that $G_{1}$ is 2-connected. But then the only possible vertex of degree two of $G_{1}$ is $v$. Thus $G_{1}$ satisfies (P1)-(P5), contradicting the minimality of $G$.

Finally we claim that, in fact, $U=\emptyset$. Suppose $U \neq \emptyset$, so there is a unique vertex $u_{0} \in U$ and $u_{0}$ has neighbours $v_{1}, v_{2}$ where $v_{1} v_{2} \in E$. If for some $i \in[2] \operatorname{deg}\left(v_{i}\right) \geq 4$, then $G \backslash\left\{u_{0}\right\}$ satisfies (P1)-(P5). Otherwise for each $i \in[2] \operatorname{deg}\left(v_{i}\right)=3$ and by the 2-connectivity of $G, u_{0}$ is the only common neighbour of $v_{1}$ and $v_{2}$, so $G \backslash\left\{u_{0}\right\} /\left\{v_{1} v_{2}\right\}$ satisfies (P1)-(P5). Either way there is a contradiction with the minimality of $G$. Thus $U=\emptyset$. But, by a result of Dirac [8], every 2-connected graph with minimum degree three has a $K_{4}$ minor, finishing the proof.

Lemma 11. Let $(D, \Gamma, R, r)$ be an irreducible representation of an mni clutter $\mathscr{C}$ and let $G$ be the underlying graph of $D=(V, A)$. Then $G$ satisfies (P1)-(P5) for some $U \subseteq V(G)$.

Proof. In a digraph, a pair of arcs $u v$ and $v u$ is said to form a digon. Two arcs are parallel if they have the same tail and the same head. For $v \in V(G)$ we denote by $\operatorname{deg}_{G}(v)$ the degree of $v$ in $G$. As we replace each parallel class by a single edge when we go from $D$ to $G, \operatorname{deg}_{G}(v)$ may be smaller than $\left|\delta^{+}(v)\right|+\left|\delta^{-}(v)\right|$. Observe that since $(D, \Gamma, R, r)$ is irreducible, we cannot contract or delete an inactive arc without changing the clutter of Steiner cuts (or equivalently the clutter of Steiner trees).

Claim 1. There are no parallel arcs in D.

Proof. Let $a, b$ be parallel arcs. If $a$ is inactive then $b$ does not appear in any Steiner cut, so $b$ is inactive and it can be deleted, without changing the clutter of Steiner cuts, a contradiction. If both $a$ and $b$ are active, we contradict (F2) as every Steiner cut containing $a$ also contains $b$. $\diamond$

Claim 2. $r$ is not incident to an inactive arc of $(D, \Gamma)$

Proof. This follows from the fact that deleting the arcs in $\delta^{+}(r)$ and contracting the arcs in $\delta^{-}(r) \cap(A-\Gamma)$ does not change the clutter of Steiner trees (choose $r$ to remain the root after contraction).

Claim 3. G has minimum degree at least two.

Proof. Suppose $\operatorname{deg}_{G}(v)=1$. If $v=r$ then by Claim $1, \delta^{-}(v)=\{f\}$ and by Claim $2, f \in \Gamma$, so every Steiner tree uses $f$, contradicting (F2). Hence $v \in V-\{r\}$. Since $(D, \Gamma, R, r)$ is an irreducible representation, every Steiner vertex must appear in a Steiner tree as a non-leaf vertex (otherwise delete the vertex and reduce the representation), so $v \in R-\{r\}$. By Claim 1 there is a unique arc $e \in \delta^{+}(v)$, and every Steiner tree uses $e$. By (F2) $e$ is inactive, but then contracting $e$ reduces the representation, a contradiction.

Claim 4. If two arcs of $(D, \Gamma)$ form a digon then one of the arcs is active. 
Proof. Otherwise we can contract the digon without changing the clutter of Steiner cuts.

Claim 5. If $\operatorname{deg}_{G}(v)=2$ then $v$ is a terminal.

Proof. Assume for a contradiction that $v$ is a Steiner vertex and let $z_{1}, z_{2}$ denote the neighbours of $v$ in $G$. Since $v$ is a Steiner vertex, $\delta^{-}(v) \neq \emptyset$.

Suppose we have $a=z_{1} v$ that is active. Every Steiner tree using $a$ must use an arc in $\delta^{+}(v)$, so $b^{\prime}=v z_{2}$ exists. Because of Claim 1 every Steiner tree using $a$ also uses $b^{\prime}$. By (F2) $b^{\prime}$ must be inactive.

We claim there is an arc $a^{\prime}=v z_{1}$. This is clear if there $z_{2} v$ is an arc. Otherwise, since contracting $b^{\prime}$ changes the clutter of Steiner trees and since $z_{2} v$ is not an arc, contracting $b^{\prime}$ must introduce new Steiner trees, and for this to happen, $a^{\prime}=v z_{1}$ has to exist.

Since $a^{\prime}$ cannot be deleted, there exists a Steiner tree $T$ that uses $a^{\prime}$. Since $v$ is a Steiner vertex, $T$ must use some arc $b=z_{2} v$. Since $b^{\prime}$ is inactive, $b$ must be active because of Claim 4 . Every Steiner tree using $b$ must use $a^{\prime}$. It follows by (F2) that $a^{\prime}$ is inactive. Hence, $a=z_{1} v, b=z_{2} v$ are active and $a^{\prime}=v z_{1}, b^{\prime}=v z_{2}$ are inactive. Then replace $a, b, a^{\prime}, b^{\prime}$ by $\operatorname{arcs} a=z_{1} z_{2}$ and $b=z_{2} z_{1}$ and remove $v$. The resulting representation $\left(D^{\prime}, \Gamma, R, r\right)$ has the same clutter of Steiner cuts as $(D, \Gamma, R, r)$. Moreover, the underlying graph of $D^{\prime}$ is a proper minor of the underlying graph of $D$. Hence, $(D, \Gamma, R, r)$ is not irreducible, which is not the case.

We therefore have $a^{\prime}=z_{1} v$ that is inactive. Contracting $a^{\prime}$ must change the clutter of Steiner trees. Hence, there exists an $\operatorname{arc} b^{\prime}=z_{2} v$. We may assume $b^{\prime}$ is inactive otherwise we are in the previous case. Since $b^{\prime}$ cannot be deleted, there exists a Steiner tree $T$ that uses $b^{\prime}$. Also, $T$ has to use some arc $a=v z_{1}$. Because of Claim $4 a$ is active. Similarly, by interchanging the roles of $a^{\prime}$ and $b^{\prime}$, there exists an active arc $b=v z_{2}$. Hence, $a=v z_{1}, b=v z_{2}$ are active and $a^{\prime}=z_{1} v, b^{\prime}=z_{2} v$ are inactive. Then replace $a, b, a^{\prime}, b^{\prime}$ by $\operatorname{arcs} a=z_{2} z_{1}$ and $b=z_{1} z_{2}$ and remove $v$. As previously, this shows that $(D, \Gamma, R, r)$ is not irreducible, a contradiction.

Claim 6. If $v$ is a terminal other than the root $r$ and $v$ has exactly two neighbours in $G$, say $z_{1}, z_{2}$, then $\delta^{+}(v)=\{a, b\}$ where $a=v z_{1}, b=v z_{2}$ are inactive.

Proof. Note first that $\delta^{+}(v) \neq \emptyset$ as $v \neq r$ is used in every Steiner tree.

Suppose that there exists an active arc $a \in \delta^{+}(v)$, say $a=v z_{1}$. By Claim 1 there is no arc parallel to $a$. By (F2) not every Steiner tree uses $a$, so there exists $b=v z_{2}$. By (F4) $\{a, b\}$ is not a transversal of the (minimum) Steiner trees, so $b$ must be inactive. By (F3) there exist distinct $i, j \in[m]$ such that,

$$
C_{i} \cap C_{j}=T_{i} \cap C_{j}=\{a\} .
$$

Since $v \in U_{i} \cap U_{j} \cap R$, Lemma 7 implies that $U_{i}, U_{j}$ are laminar, say $U_{i} \subseteq U_{j}$. By (F1) there exists $e \in\left(C_{i} \cap T_{i}\right)-\{a\}$ with head $w$. By $(\ddagger) e \notin C_{j}$, so $w \in U_{j}-U_{i}$. Since $e \in T_{i}$, there is a dipath connecting $w$ to the root $r$ in $T_{i} \cup(A-\Gamma)$. It follows by $(\ddagger)$ that this dipath must contain a $w v$-dipath in $D\left[U_{j}\right]$. So there is an arc $b^{\prime}$ in $D\left[U_{j}\right]$ with head $v$. Since $\operatorname{deg}_{G}(v)=2,\left\{b, b^{\prime}\right\}$ is a digon. It follows by Claim 4 that $b^{\prime}$ is active. But then every Steiner tree using $b^{\prime}$ also uses $a$, contradicting (F2). 
Thus all arcs in $\delta^{+}(v)$ are inactive. Furthermore, if there is just one such arc then we can reduce the representation by contracting the arc. The result now follows by Claim 1 .

(P1) holds trivially. Let $U$ be the set of vertices of degree 2 of $G$, and if $r \in U$ then let $u_{0}=r$, otherwise when $U \neq \emptyset$, choose arbitrarily a $u_{0} \in U$. (P2) follows by Claim 3. (P3) Suppose otherwise, i.e. there are neighbouring vertices $u, v$ of degree two in $G$. By Claim $5 u, v$ are both terminals, and by Claims 6 and 2, none of $u, v$ is the root. But then, Claim 6 implies that there are inactive arcs $u v$ and $v u$, contradicting Claim 4. (P4) Suppose otherwise, i.e. there exists $v \in V-\{r\}$ that has exactly two neighbours $z_{1}, z_{2}$ in $G$ and there is an $\operatorname{arc} c=z_{1} z_{2}$. By Claim 5 $v$ is a terminal and by Claim 6 we have inactive $\operatorname{arcs} a=v z_{1}, b=v z_{2}$. If $c$ is active, then replace $c$ by $c:=z_{1} v$, and if $c$ is inactive, then contract $a=v z_{1}$ (the vertex corresponding to $v z_{1}$ becomes a terminal). If an $\operatorname{arc} d=z_{2} z_{1}$ also exists, then modify the representation similarly. The resulting representation $\left(D^{\prime}, \Gamma, R^{\prime}, r\right)$ has the same clutter of Steiner cuts as $(D, \Gamma, R, r)$. Moreover by Claim 1 the underlying graph of $D^{\prime}$ is a proper minor of $D$. Hence, $(D, \Gamma, R, r)$ is reducible, a contradiction. (P5) Suppose otherwise, i.e. there exist $v_{1}, v_{2} \in V-\{r\}$ and both have exactly two neighbours that are the same, say $z_{1}, z_{2}$. By Claim $5 v_{1}, v_{2}$ are terminals and by Claim 6 we have inactive arcs $v_{1} z_{1}, v_{1} z_{2}, v_{2} z_{1}, v_{2} z_{2}$. By Claims 1 and 4 and (P4), all other arcs with both tail and head in $\left\{v_{1}, v_{2}, z_{1}, z_{2}\right\}$ are active $\operatorname{arcs} z_{i} v_{j}$ for some $i, j \in[2]$. Note further that for $i \in[2]$, we cannot simultaneously have active arcs $z_{i} v_{1}$ and $z_{i} v_{2}$, due to (F2). Let $D^{\prime}$ be obtained from $D$ by identifying $v_{1}, v_{2}$ followed by deleting $\left\{v_{2} z_{1}, v_{2} z_{2}\right\}$. The resulting representation $\left(D^{\prime}, \Gamma, R^{\prime}, r\right)$ has the same clutter of Steiner cuts as $(D, \Gamma, R, r)$. Moreover, the underlying graph of $D^{\prime}$ is a proper minor of the underlying graph of $D$. Hence, $(D, \Gamma, R, r)$ is reducible, a contradiction.

We are now ready to prove Theorem 6 , part (iv'), stating that $D$ is not series-parallel.

Proof of Theorem 6, part ( $\left.i v^{\prime}\right)$. Let $(D, \Gamma, R, r)$ be an arbitrary representation of $\mathscr{C}$. Then $(D, \Gamma, R, r)$ is reducible to an irreducible representation $\left(D^{\prime}, \Gamma^{\prime}, R^{\prime}, r^{\prime}\right)$. Let $G$ (resp. $\left.G^{\prime}\right)$ be the underlying graph of $D$ (resp. $\left.D^{\prime}\right)$. By Lemma 11, $G^{\prime}$ satisfies (P1)-(P5). It follows from

Lemma 10 that $G^{\prime}$ has a $K_{4}$ minor. By definition of reducibility, $G^{\prime}$ is a minor of $G$, hence $G$ has a $K_{4}$ minor. Thus $G$, and therefore $D$, is not series-parallel.

\section{Concluding remarks}

We conclude by providing evidence that a characterization of the idealness of the clutter of Steiner cuts is likely to remain elusive. We observed in $\S 3$ that $\mathscr{C}=\{\{1,2\},\{1,3\},\{2,3\}\}$ is not the clutter of Steiner cuts of any digraph. However, $\mathscr{C}$ is the clutter of Steiner cuts of a marked digraph. In fact,

Proposition 12. Every clutter is the clutter of Steiner cuts of a marked digraph.

Proof. Consider an arbitrary clutter $\mathscr{C}$. Construct a marked digraph $(D, \Gamma)$ with terminals $R$ and root $r$ as follows: for each $e \in E(\mathscr{C})$ we have a Steiner vertex $v_{e}$ and an active arc $v_{e} r$; for 
each $C \in \mathscr{C}$ we have a terminal $u_{C}$; and for all $C \in \mathscr{C}$ and $e \in C$ we have an inactive arc $u_{C} v_{e}$. (See Figure 3 for an example.) It can be readily checked that $\mathscr{C}$ is the clutter of Steiner cuts of the representation $(D, \Gamma, R, r)$.

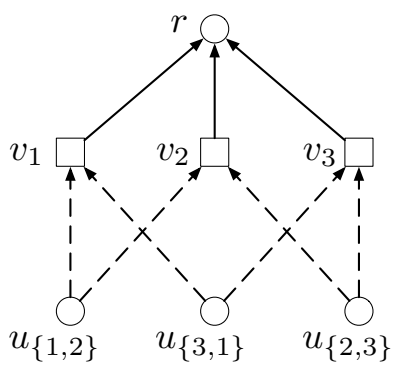

Figure 3: Construction of $\{\{1,2\},\{2,3\},\{3,1\}\}$ as the clutter of Steiner cuts of a marked digraph. Round vertices are terminals, solid arcs are active.

Characterizing when an arbitrary clutter is ideal has shown to be very difficult [7]. In light of the previous proposition, so is characterizing the idealness of clutters of Steiner cuts of marked digraphs. Therefore, a more promising line of investigation is to find sufficient conditions for such a clutter to be ideal as is done in Theorem 1 .

\section{Bibliography}

[1] Bondy, J.A. and Murty U.S.R.: Graph theory. Springer (2007)

[2] Bridges, W.G. and Ryser H.J.: Combinatorial designs and related systems. J. Algebra 13, 432-446 (1969)

[3] Charikar, M., Chekuri, C., Cheung, T., Dai, Z., Goel, A., Guha, S., Li, M.: Approximation algorithms for directed Steiner problems. J. of Algorithms 33, 73-91 (1999)

[4] Chopra, S. and Rao, M.R.: The Steiner tree problem I: formulations, compositions and extension of facets. Math. Program. 64, 209-229 (1994)

[5] Conforti, M., Cornuéjols, G., Zambelli, G.: Integer programming. Springer (2014)

[6] Cornuéjols, G., Guenin, B., Margot, F.: The packing property. Math. Program. Ser. A 89, 113-126 (2000)

[7] Cornuéjols, G., Guenin, B., Tunçel, L.: Lehman matrices. J. Combin. Theory Ser. B 99, $531-556(2009)$

[8] Dirac, G.A.: A property of 4-chromatic graphs and some remarks on critical graphs. J. London Math. Soc. 27, 85-92 (1952)

[9] Duffin, R.J.: Topology of series-parallel networks. J. Math. Anal. Appl. 10, 303-318 (1965) 
[10] Edmonds, J.: Optimum branchings. J. Res. Nat. Bur. Standards Sect. B 71B(12), 233-240 (1967)

[11] Edmonds, J. and Fulkerson, D.R.: Bottleneck extrema. J. Combin. Theory Ser. B 8, 299-306 (1970)

[12] Ford, L.R. and Fulkerson, D.R.: Maximal flow through a network. Canadian J. Math. 8, 399-404 (1956)

[13] Friggstad, Z., Könemann, J., Kun-Ko, A.L., Shadravan, M., Tulsiani, M.: Linear programming hierarchies suffice for directed Steiner tree. Proceedings, Integer Programming and Combinatorial Optimization, Lecture Notes in Computer Science: 285-296 (2014)

[14] Fulkerson, D.R.: Blocking and anti-blocking pairs of polyhedra. Math. Programming 1, 168-194 (1971)

[15] Fulkerson, D.R.: Packing rooted directed cuts in a weighted directed graph. Math. Programming 6, 1-13 (1974)

[16] Goemans, M.X.: Arborescence polytopes for series-parallel graphs. Discrete Appl. Math. 51, 277-289 (1994)

[17] Guenin, B.: A characterization of weakly bipartite graphs. J. Combin. Theory Ser. B 83, 112-168 (2001)

[18] Halperin, E. and Krauthgamer, R.: Polylogarithmic inapproximability. Proceedings of ACM Symposium on Theory of Computing, 585-594 (2003)

[19] Isbell, J.R.: A class of simple games. Duke Math. J. 25(3), 423-439 (1958)

[20] Lasserre, J.: An explicit exact SDP relaxation for nonlinear 0-1 programs. Proceedings, Integer Programming and Combinatorial Optimization, Lecture Notes in Computer Science: 293-303 (2001)

[21] Lasserre, J.: Global optimization with polynomials and the problem of moments. SIAM J. Optimization 11(3), 796-817 (2001)

[22] Lehman, A.: A solution of the Shannon switching game. Society for Industrial Appl. Math. 12(4), 687-725 (1964)

[23] Lehman, A.: On the width-length inequality. Math. Program. 17(1), 403-417 (1979)

[24] Lehman, A.: The width-length inequality and degenerate projective planes. In "Polyhedral Combinatorics" (W. Cook and P.D. Seymour, Eds.), DIMACS Series in Discrete Math. and Theoretical Computer Science, Vol. I, 101-105 (1990)

[25] Lovász, L. and Schrijver, A.: Cones of matrices and set-functions and 0-1 optimization. SIAM J. Optimization 1, 166 -190 (1991)

[26] Prodon, A., Liebling, T.M., Gröflin, H.: Steiner's problem on 2-trees. Research Report RO 850315, Ecole Polytechnique de Lausanne (1985) 
[27] Rothvoß, T.: Directed Steiner tree and the Lasserre hierarchy. CoRR, vol. abs/1111.5473 (2011)

[28] Schaffers, M.: Network flow design III. Polyhedral characterization of the single source fixed costs problem on series-parallel graphs. CORE Discussion Paper, Université Catholique de Louvain, Louvain (1991)

[29] Schrijver, A.: Combinatorial optimization. Polyhedra and efficiency. Springer (2003)

[30] Seymour, P.D.: On Lehman's width-length characterization. In "Polyhedral Combinatorics" (W. Cook and P.D. Seymour, Eds.), DIMACS Series in Discrete Math. and Theoretical Computer Science, Vol. I, 107-117 (1990)

[31] Seymour, P.D.: The forbidden minors of binary matrices. J. London Math. Society 2(12), 356-360 (1976)

[32] Seymour, P.D.: The matroids with the max-flow min-cut property. J. Combin. Theory Ser. B 23, 189-222 (1977)

[33] Shepherd, F.B.: Applying Lehman's theorems to packing problems. Math. Program. 71, 353-367 (1995)

[34] Sherali, H. and Adams, W.: A hierarchy of relaxation between the continuous and convex hull representations. SIAM J. Discrete Math. 3, 411 -430 (1990)

[35] Zosin, L. and Khuller, S.: On directed Steiner trees. Proceedings of ACM-SIAM Symposium on Discrete Algorithms, 59-63 (2002) 